\title{
Neue Entwicklungen zu den KN
}

\section{Dennis Edler ${ }^{1}$}

Accepted: 8 February 2021 / Published online: 4 March 2021

(c) The Author(s) 2021

Liebe Leserinnen und Leser,

Mit dem neuen Jahr 2021 und zugleich mit dem 71. Jahrgang freue ich mich, die Hauptschriftleitung der Kartographischen Nachrichten übernehmen zu dürfen. Die Leistung meiner Vorgänger, die bekannteste und traditionsreichste Fachzeitschrift der Kartographie im deutschsprachigen Raum über sieben Jahrzehnte so erfolgreich geleitet und weiterentwickelt zu haben, verdient allergrößten Respekt!

Was die KN als Fachzeitschrift auszeichnet, ist die enge Einbindung der aktiven Mitglieder der DGfK, aber auch der ÖKK und SGK. Dies wird durch die Beiträge im Info- und Praxisteil in jeder quartalsweisen Ausgabe deutlich. Dieser Austausch zu stets aktuellen Entwicklungen und Themen aus dem weiten Spektrum der Kartographie und Geoinformationswissenschaften sowie zu Vereinsaktivitäten, bspw. in Sektionen und Kommissionen der DGfK, steht für Mitteilungen am „Puls der Zeit“ der Fachdisziplin und des Vereinslebens. Daher bildet der Info- und Praxisteil einen unverzichtbaren Teil, der die KN, auch im Vergleich mit anderen Fachzeitschriften der Kartographie, als Zeitschrift des Austausches und der Teilhabe-über den Tellerrand der Wissenschaft hinaus-hervorhebt.

Über einen „lebendigen“ und inhaltsreichen Info- und Praxisteil hinaus wird jedoch die Zukunft der KN insbesondere dadurch bestimmt, wie sich die Zeitschrift im internationalen Wettbewerb behauptet. Aktuelle englischsprachige Publikationen in einflussreichen und sichtbaren Zeitschriften werden von Wissenschaftlerinnen und Wissenschaftlern erwartet, um als Teil der „Forschungsfront“ akzeptiert zu werden. Auch bei der Vergabe von Forschungsprojekten und -geldern sowie beim Abschluss von Qualifikationsvorhaben an Hochschulen (v.a. Promotionen) werden die Reputationen der Zeitschriften, in denen jüngst Publikationen platziert wurden, berücksichtigt. Entsprechend hoch ist der

Dennis Edler

Dennis.Edler@ruhr-uni-bochum.de

1 Bochum, Germany
Wettbewerb zwischen Fachzeitschriften, die Anforderungskriterien aus der Wissenschaft erfüllen zu können. Diesen Entwicklungen und Erwartungen stellen sich die KN:

Dank der international ausgerichteten Strukturen des aktuellen Verlagshauses (Springer Nature) sind die wissenschaftlichen Artikel seit 2019 erheblich sichtbarer und werden in der internationalen Community-so zeigen es die Zitationen der einzelnen Beiträge auf der KN-Webseite-berücksichtigt. Durch das Projekt DEAL haben Hochschulen und zahlreiche Forschungsinstitute in Deutschland und einigen Partnerländern Zugriff auf zugangsfreie (OpenAccess) Veröffentlichungsoptionen in den Zeitschriften des Springer-Verlags-und damit auch in den KN. Entsprechend kann die KN die Sichtbarkeit der ,OpenAccess-Jahre' 2019 und 2020 weiter aufrechthalten und ihre wissenschaftlichen Beiträge der internationalen und englischsprachigen Community weiter gut zugänglich machen. Folgen mehr Zitationen der Zeitschrift in renommierteren Zeitschriften-mit „Journal Impact Factor“ (JIF)-formiert sich ein wichtiger Baustein, damit die KN in die Reihe der internationalen Fachzeitschriften mit JIF aufgenommen werden kann. Um dies zu erreichen, ist die ganze wissenschaftliche Community der Kartographie in Deutschland, Österreich, der Schweiz und weiterer Länder gefragt, Manuskripte mit hoher Qualität und Zitationspotenzial in den KN einzureichen. An dieser Stelle sei ebenfalls auf die Präsidenten-Kolumne dieser Ausgabe verwiesen.

Die Ziele der KN, sowohl den Info- und Praxisteil als auch den wissenschaftlichen Teil dauerhaft in hoher Qualität herauszugeben, erfordert ein Umdenken in der Struktur und Aufgabenverteilung der ehrenamtlich tätigen Schriftleitung. Dank der großen Bereitschaft von Dr. Jana Moser (LeibnizInstitut für Länderkunde-Leipzig) wird der Info- und Praxisteil seit diesem Heft durch eine eigene Position in der KNSchriftleitung (Co-Editor Info and Practice) betreut. Neben den inhaltlichen Rubrikverantwortlichen (s. weiterführend S. A-43) unterstützen dabei ein professionelles Lektorat und Layout.

Die wissenschaftlichen Beiträge der ersten Ausgabe des 71. KN-Jahrgangs verdeutlichen, dass die Zeitschrift 
weiterhin ein breites inhaltliches Spektrum der Kartographie abdeckt. Die sechs Artikel von Autorinnen und Autoren vieler Forschungsstandorte (in Rostock, Berlin, Tübingen, Poznań, Graz, Mexico City, Guadalajara und Bochum) bedienen eine Themenvielfalt von der Historischen Kartographie, über die Themakartographie (einschl. theorieorientierter Zugänge der Geographie), bis hin zu aktuellen Entwicklungen zur Konstruktion 3D-kartographischer Medien.

Im Namen der gesamten neuen Schriftleitung wünsche ich Ihnen viel Freude beim Lesen!

Herzliche Grüße
Funding Open Access funding enabled and organized by Projekt DEAL.

Open Access This article is licensed under a Creative Commons Attribution 4.0 International License, which permits use, sharing, adaptation, distribution and reproduction in any medium or format, as long as you give appropriate credit to the original author(s) and the source, provide a link to the Creative Commons licence, and indicate if changes were made. The images or other third party material in this article are included in the article's Creative Commons licence, unless indicated otherwise in a credit line to the material. If material is not included in the article's Creative Commons licence and your intended use is not permitted by statutory regulation or exceeds the permitted use, you will need to obtain permission directly from the copyright holder. To view a copy of this licence, visit http://creativecommons.org/licenses/by/4.0/.

Dennis Edler

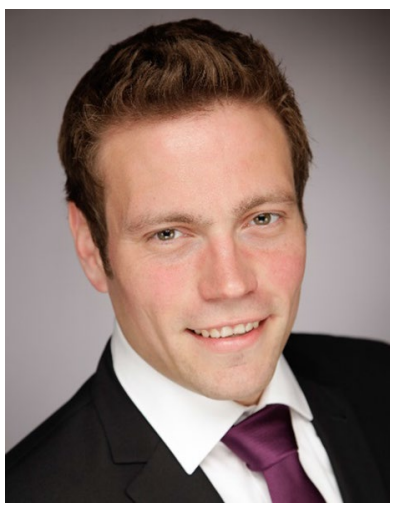

